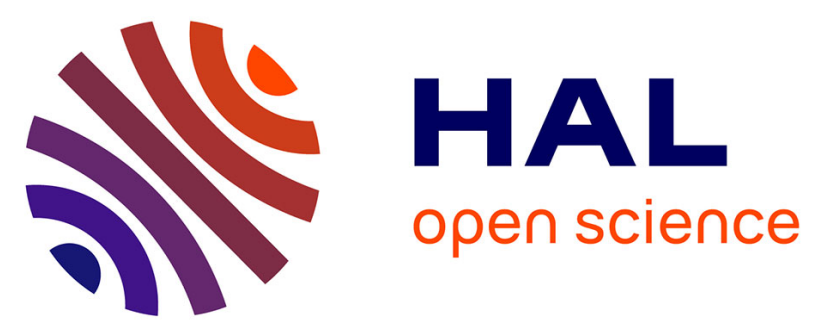

\title{
A saw is first identified as an object used on wood: ERP evidence for temporal differences between Thematic and Functional similarity relations
}

\author{
Yannick Wamain, Ewa Pluciennicka, Solène Kalénine
}

\section{- To cite this version:}

Yannick Wamain, Ewa Pluciennicka, Solène Kalénine. A saw is first identified as an object used on wood: ERP evidence for temporal differences between Thematic and Functional similarity relations. Neuropsychologia, 2015, 71 (4), pp.28-37. 10.1016/j.neuropsychologia.2015.02.034 . hal-02109761

\section{HAL Id: hal-02109761 \\ https://hal.science/hal-02109761}

Submitted on 5 Jan 2022

HAL is a multi-disciplinary open access archive for the deposit and dissemination of scientific research documents, whether they are published or not. The documents may come from teaching and research institutions in France or abroad, or from public or private research centers.
L'archive ouverte pluridisciplinaire HAL, est destinée au dépôt et à la diffusion de documents scientifiques de niveau recherche, publiés ou non, émanant des établissements d'enseignement et de recherche français ou étrangers, des laboratoires publics ou privés. 
Title : A saw is first identified as an object used on wood: ERP Evidence for temporal differences between Thematic and Functional similarity relations

Author names and affiliations : Yannick Wamain ${ }^{1,2}$, Ewa Pluciennicka ${ }^{1,2}$, Solène Kalénine ${ }^{1,3,4}$

${ }^{1}$ Université Lille Nord de France, F-59000 Lille, France

${ }^{2}$ UDL3, URECA, F-59653 Villeneuve d'Ascq Cedex, France

${ }^{3}$ UDL3, IRHIS, F-59653 Villeneuve d'Ascq Cedex, France

${ }^{4}$ CNRS, URM8529, F-59653 Villeneuve d'Ascq Cedex, France.

\section{Corresponding author:}

Yannick WAMAIN

Mail : ywamain@gmail.com

Phone : +33 320416953

Laboratoire URECA

Batiment A2 - Niveau forum +2

Université Charles-de-Gaulle Lille III

Domaine universitaire du Pont de Bois, BP 149

59653 Villeneuve d'Ascq Cedex, France 


\title{
A saw is first identified as an object used on wood: ERP Evidence for temporal differences between Thematic and Functional similarity relations
}

\author{
Yannick Wamain ${ }^{1,2}$, Ewa Pluciennicka ${ }^{1,2}$, Solène Kalénine ${ }^{1,3,4}$ \\ ${ }^{1}$ Université Lille Nord de France, F-59000 Lille, France \\ ${ }^{2}$ UDL3, URECA, F-59653 Villeneuve d'Ascq Cedex, France \\ ${ }^{3}$ UDL3, IRHIS, F-59653 Villeneuve d'Ascq Cedex, France \\ ${ }^{4}$ CNRS, URM8529, F-59653 Villeneuve d'Ascq Cedex, France.
}

\begin{abstract}
The role of functional and motor information in manipulable artifact object semantic organization is still poorly understood. In particular, several types of semantic relations involving object functional knowledge may be distinguished. Functional similarity relations group objects with similar functions at relatively specific (e.g. saw-axe, both used to cut wood) or general (saw-knife, both used to cut) levels. Thematic relations group objects based on their complementarity in events (saw used upon/with wood). Recent eye-tracking data showed distinct temporal time courses for the different semantic relations, with fastest processing of thematic relations and slowest processing of general function similarity relations. Behavioral data suggest the involvement of distinct cognitive mechanisms in manipulable artifact object semantic processing. The aim of the present study was to assess the neural correlates of thematic, and specific and general function similarity relation processing. Specifically, we investigated whether time course differences between semantic relations could be highlighted at the neurophysiological level. We used a protocol combining semantic priming with electroencephalography, and manipulated the type of semantic relation and the duration of the interval between prime and target objects. Two consistent and complementary results were shown. On N1 and P3 components, semantic priming was observed for thematic relations only. On N400 component, the type of semantic relation interacted with interval duration, and semantic priming was visible for all 3 relations after the longest interval only. Results revealed graded processing time courses for thematic, specific function similarity, and general function similarity relations at the neural level, and further indicate that thematic relations impact object processing during the early stages of object recognition. Findings suggest a hierarchical organization of three types of semantic relations based on functional knowledge. The parallel between semantic relations involving manipulable artifact objects and levels of action representations is discussed.
\end{abstract}

\section{Keywords:}

ERP, Semantic Priming, Thematic and Functional Knowledge, Manipulable artifacts. 


\section{Introduction}

After decades of research on semantic categories, there is still little known about the organization of our knowledge about manipulable artifact objects. Object semantic categories can be viewed as classes of objects with similar features (Rogers \& McClelland, 2004). Importantly, the relative weight of perceptual (e.g., visual, olfactory) and non-perceptual (e.g., functional) features in the composition of object categories may vary depending on object kind (Farah \& McClelland, 1991; Warrington \& Shallice, 1984). By contrast with natural object categories, functional and/or motor features appear particularly relevant for artifact object categories as witnessed, for instance, by the greater proportion of functional/motor features generated in response to artifact concept names in comparison to natural object names (Cree \& McRae, 2003; Garrard, Ralph, Hodges, \& Patterson, 2001; McRae, Cree, Seidenberg, \& McNorgan, 2005). Yet the role of the different aspects of functional/motor information in object semantic categories is still relatively unclear.

Many behavioral studies reported an influence of motor feature similarity (i.e. similarity in manipulation) on object semantic processing (Helbig, Graf, \& Kiefer, 2006; Labeye, Oker, Badard, \& Versace, 2008; Myung, Blumstein, \& Sedivy, 2006; Watson \& Buxbaum, 2014). In priming paradigms, participants are faster to make a lexical decision about a target word when it was preceded by a related prime that shared the same manipulation as the target (Labeye et al., 2008; Myung et al., 2006). During target object visual search among distractors, participants tend to look longer at distractor objects used in a same way as the target in comparison to unrelated distractors (Lee, Middleton, Mirman, Kalénine, \& Buxbaum, 2013; Myung et al., 2006). Similarly, using a blocked cyclic word-picture matching task known to be sensitive to semantic interference, Watson and Buxbaum (2014) showed poorer performance during blocks containing 
tools used with similar versus dissimilar action, the amount of interference being function of the magnitude of manipulation similarity between objects. Manipulation similarity also influences object conceptual processing at the neurophysiological level (Kiefer, Sim, Helbig, \& Graf, 2011). Using a priming paradigm, Kiefer et al. demonstrated reduced P1 and N400 components when target and prime objects afforded similar actions in comparison to dissimilar actions. Taken together, these findings suggest that manipulation similarity shapes, at least to a certain extent, object semantic structure at both the cognitive and neurophysiological levels.

The role of functional relations between objects in semantic organization is somehow less understood. Although frequently considered as a whole in property generation studies, dissociations between object manipulation and functional knowledge have been documented in several behavioral and neuroimaging studies (Boronat et al., 2005; Buxbaum and Saffran, 2002; Canessa et al., 2008; Garcea \& Mahon, 2012; Kellenbach et al., 2003). In Buxbaum \& Saffran (2002), apraxic patients showed impaired manipulation knowledge but relatively preserved functional knowledge, whereas nonapraxics tended to exhibit the opposite pattern. Neuroimaging findings highlighted differences in the neural networks specifically involved during manipulation and functional knowledge processing, in the left fronto-parietal cortex and anterior temporal lobe, respectively (Boronat et al., 2005; Canessa et al., 2008; Kellenbach et al., 2003). Overall, this suggests that the role of functional knowledge in object conceptual organization cannot be generalized from what is known about the influence of manipulation relations between objects.

Moreover, functional knowledge may impact semantic categories in different ways. On the one hand, similarity between functional features may be one important determinant of object semantic structure. For instance, screw, clamp, and hammer are semantically related by virtue of common functional features such as "used for carpentry" (McRae et al., 2005). Functional 
similarity can further be defined at different levels of generality. Screw is functionally similar to both clamp and hammer if the functional feature considered is "used for carpentry", but screw is only similar to clamp if the function considered is more specific, for example "used for holding things together". On the other hand, semantic relations based on functional knowledge can be also expressed in terms of complementarity between objects. Thematic relations bound objects that "perform complementary roles in the same scenario or event" (Estes, Golonka, \& Jones, 2011). In the case of manipulable artifact objects, thematic relations often refer to objects that are directly used together (i.e. screwdriver-screw, saw-wood). Therefore, thematically-related objects can be considered functionally related in the broad sense, although not typically functionally similar.

There is growing evidence indicating that thematic knowledge could impact object semantic organization as well as feature similarity (Estes et al., 2011; Estes \& Jones, 2009; Hare, Jones, Thomson, Kelly, \& McRae, 2009; Kalénine \& Bonthoux, 2008; Kalénine et al., 2009; Lin \& Murphy, 2001; Mirman \& Graziano, 2012; Wisniewski \& Bassok, 1999). Several studies demonstrated priming effects between thematically related word pairs (e.g., knife-steak) (Estes \& Jones, 2009; Hare et al., 2009; Moss, Ostrin, Tyler, \& Marslen-Wilson, 1995). Interestingly, in the case of manipulable artifacts, thematic relations appeared to be more quickly identified than other semantic relations based on feature similarity (Kalénine \& Bonthoux, 2008; Kalénine et al., 2009). Growing evidence coming from fMRI and patient studies indicates that the posterior temporo-parietal cortex is involved in thematic relation processing (Kalénine et al., 2009; Mirman \& Graziano, 2012; Schwartz et al., 2011; Tsagkaridis, Watson, Jax, \& Buxbaum, 2014). The temporo-parietal cortex is an important neuroanatomical substrate of object use representations (Beauchamp \& Martin, 2007; Buxbaum, Shapiro \& Coslett, 2014; Johnson-Frey, 
2004; Vingerhoets, 2014). Accordingly, we assume that for manipulable artifacts, thematic relation processing relies, at least partially, on object use representations, that would be quickly recruited during object processing via the activity of the posterior temporo-parietal cortex. In contrast, functional similarity relation processing - in particular at the general level- may not benefit from the early activation of object use representations.

In this context, the general issue addressed in this study concerned whether semantic relations between manipulable artifacts are organized along distinct types of functional knowledge at the cognitive and neural levels. Particularly, we investigated whether processing semantic relations based on object complementarity, namely thematic relations, could be distinguished from processing semantic relations based on functional similarity. The distinction between thematic and functional similarity relations has been the focus of a few previous eyetracking studies (Kalénine, Mirman, \& Buxbaum, 2012; Kalénine, Mirman, Middleton, \& Buxbaum, 2012; Pluciennicka, Coello, \& Kalénine, submitted). In these studies, participants had to identify a target object among 4 object pictures, one of the distractor objects being semantically related to the target. Eye movements during target identification were used to measure the temporal dynamics of semantic competition between target and related distractor, and accordingly, to assess the incidental processing time course of a particular semantic relation between objects. Depending on the conditions, target and related distractor could be thematically related (Thematic condition: e.g., broom-dustpan), functionally similar at the specific level (Specific Function condition: e.g., broom-vacuum cleaner), or functionally similar at the general level (General Function condition: e.g., broom-sponge). The overall semantic relatedness was equivalent between conditions. The main findings highlighted differences in semantic processing time course between conditions. Processing of thematic relations was fast but transient, with 
competition effects rising around $500 \mathrm{~ms}$ and ending around $1000 \mathrm{~ms}$ after target onset. Processing of general functional similarity relations, in contrast, was visible later and longerlasting, with competition effects between 700 and $1200 \mathrm{~ms}$ after target onset. Processing of specific functional similarity relations was intermediate. The eye-tracking results suggest that thematic, specific functional similarity, and general functional similarity relations between objects rely (at least partially) on distinct cognitive processes. Moreover, results have been interpreted in favor of a hierarchical organization of semantic relations, with faster access to lower levels of representation more closely related to object use direct experience (i.e. thematic relations such as saw-wood may directly involve sawing), and slower access to higher levels of representation (i.e. general functional similarity relations such as saw-knife).

The specific aim of the present work was to investigate the temporal dynamics of processing of thematic, and specific and general functional similarity relations between objects at the neural level. Specifically, we tested whether the behavioral differences observed in the eyetracking experiments (Kalénine, Mirman, Middleton, et al., 2012; Pluciennicka et al., submitted) could reflect differences in the neurophysiological correlates of thematic, specific function similarity, and general functional similarity relation processing. Neural processing differences between the three types of semantic relations would provide new inputs into the question of how various kinds of functional knowledge participate to object identification and categorization. To this end, thematic, specific functional similarity relations were manipulated in a priming paradigm while EEG was recorded (see Kiefer et al., 2011 for a similar EEG paradigm).

Temporal differences between thematic, specific functional similarity and general functional similarity relation processing could have two possible consequences on the neurophysiological responses collected during semantic priming. First, thematic priming effects 
may be visible on earlier components than functional priming effects, especially at the general level. In other words, while semantic priming should be observed on the N400 component for all semantic relations (Deacon, Hewitt, Yang, \& Nagata, 2000; Eddy, Schmid, \& Holcomb, 2006; Kutas \& Iragui, 1998; McPherson \& Holcomb, 1999), priming effects for thematically related pairs may be observed on the P300, N1 and potentially P1 components (see Kiefer "early semantic priming" on P1). Although probable sources of early and late components in priming paradigms are rarely reported, N400 semantic priming-related effects have been related to the occipito-temporal cortex (sim 2014, kiefer, 2011, lau 2013, geukes 2013). Moreover, according to a few priming studies, probable sources of earlier components may also include regions of the fronto-parietal cortex (kiefer, 2011, sim, 2014, wirth 2008). Thus, one may expect both frontoparietal and occipito-temporal networks to be involved in target object semantic processing, with possible variations depending on timing.

Second, time allocated to prime processing should differently impact semantic priming effects depending on the type of relation. When the Inter-Stimulus Interval (ISI) between prime and target is short (prime duration being constant otherwise), semantic processing of the prime picture is limited and only prime properties that are quickly evoked from object pictures are already activated when the target object is displayed. Thus, after short ISI, thematically-related prime pictures would have already activated congruent thematic knowledge (e.g., "wood" quickly activates the thematic associate "saw") at the onset of target picture processing (e.g. "saw"), while functionally similar primes would not have been submitted to sufficient processing to activate congruent functional knowledge (e.g. "knife" may quickly activates irrelevant thematic associates such as fork or steak, but slowly activates the functional property "to cut"). After longer ISI, prime pictures would have been processed long enough to fully activate 
congruent functional knowledge when the target picture is delivered. In contrast, the early and transient activation of thematic knowledge from prime pictures may have started to decrease at target onset (or may be still ongoing, depending on whether thematic and functional activations are partially overlapping in time or not). Accordingly, in the short ISI condition maximal priming would be obtained for thematic relations, and priming would be reduced or absent for function similarity relations (in particular general ones that require most time to be activated), on both early and late ERP components. Priming effects for general function similarity relations should emerge in the long ISI condition and would be equal or superior to thematic priming, in particular on late ERP components. Hence, manipulating the ISI between prime and target during semantic priming in EEG appeared well suited to assess potential temporal differences in semantic processing of thematic, specific function similarity, and general function similarity relations between objects. Overall, we assumed that the temporal advantage of thematic relation processing could be related to the activity of the posterior temporo-parietal cortex underlying object use representations. 


\section{Method}

Eighteen adults (mean age 25.6; age range 19-37; 10 women) participated in the experiment and were paid 15 euros for their participation. All participants had normal or corrected-to-normal visual acuity and had French as native or primary language. Besides, participants were right-handed according to the Edinburgh handedness quotients (50-100\%; mean 90\%; Oldfield, 1971), and had no reported history of dyslexia or any neurological diseases. The experimental procedure was approved by the local ethical committee in accordance with the Helsinki declaration.

\subsection{Stimuli}

Overall, the present study involved the same materials as Pluciennicka et al.'s study (submitted). Color photographs of 15 common objects (200 x 200 pixels) were used as target objects. Each target object was associated to 6 prime objects (Figure 1 but see also the complete list of objects used in Appendix 1). Half of the prime objects were semantically related to the target, half were unrelated to the target. For related object pairs, the type of semantic relation was manipulated in three conditions: Thematic, Specific Function and General Function. In the Thematic condition, the prime object could be used to act upon/with the target (e.g., cup-spoon). In the Specific Function condition, the prime and the target objects shared the same function at a relative specific level (cup-coffee maker can both be used to drink coffee). In the General Function condition, the prime and the target objects shared the same function at a relative general level (cup-glass; can both be used to drink). Finally, for unrelated object pairs, the prime objects were neither semantically nor phonologically related to the target object. However, one of the 3 unrelated primes was chosen to be visually similar to the target object, either in shape or in color. 
Semantic relations were selected from a large property generation study (Pluciennicka, Coello, \& Kalénine, in press). The concept-property relations collected this way « are assumed to provide a window into semantic memory» (Cree and Mcrae, 2003, pp. 167). In our property generation study, we focused on artifact concept names and properties related object utilization. Properties were prompted with sentences such as "[NAME] can be used with/upon [BLANK]" and "[NAME] can be used to [BLANK]". Thematic relations were defined as object pairs that regularly appear together in the "[NAME] can be used with/upon [BLANK]" sentences in participants' productions. Functional similarity relations were defined as two concepts that received a similar response after "[NAME] can be used to [BLANK]" prompts. Note that functional similarity relations that also appeared thematically related in the generation task were excluded. Objects functionally similar at the specific level were also related at the more general level (e.g. "can be used to drink" has been generated in response to cup, coffee maker, and glass), but objects functionally similar at the general level were not similar at the specific level (e.g. "can be used to drink coffee" has been only generated in response to cup and coffee maker, not glass).

Additional ratings were collected on picture pairs in order to control for visual similarity, manipulation similarity, and semantic relatedness (see Pluciennicka et al. (submitted). Visual and manipulation similarity between target pictures and their corresponding related and unrelated primes ${ }^{1}$ were evaluated using a 7-point Likert scale. Twelve additional participants (who did not participate in the present experiment) were asked to rate to what extend two objects of a pair 1) were visually similar 2) could be manipulated in the same way. The 3 types of semantic relations

\footnotetext{
${ }^{1}$ The number of semantically unrelated and visually dissimilar prime pictures has been doubled in the present experiment in comparison to Pluciennicka et al. submitted. The norms reported here only concern the initial set of unrelated primes. However, the second set has only been added to keep the design balanced and has not been included in the analyses.
} 
were overall equivalent in terms of visual similarity $[\mathrm{F}(2,42)=1.79, \mathrm{p}=.18]$. Semantically unrelated but visually similar primes were judged more visually similar to the target than visually dissimilar unrelated primes $[\mathrm{F}(1,28)=16.61, \mathrm{p}<.001]$, but were equivalent to semantically related objects in terms of visual similarity $[\mathrm{F}(1,58)=0.05, \mathrm{p}=.82]$. Similarly, the 3 types of semantic relations were overall equivalent in terms of manipulation similarity $[F(2,42)=2.39$, $\mathrm{p}=.10]$. Indices of overall semantic relatedness were based on Latent Semantic Analysis (LSA) measures extracted from text corpora. Overall semantic relatedness was greater for semantically related object pairs than unrelated object pairs $[\mathrm{F}(1,73)=86.94, \mathrm{p}<.001]$, but equivalent between thematic, specific function, and general function relations $[\mathrm{F}(2,42)=1.28, \mathrm{p}=.29]$. In other words, we could ensure that object pairs in the 3 semantic conditions were equally but differently related. Mean norming values from Pluciennicka et al. (submitted) are presented in Table 1.

Table 1: Mean values and standard deviations of normative ratings and LSA measures for the thematic, specific function and general function related and unrelated object pairs.

\begin{tabular}{lccc}
\hline \multicolumn{1}{c}{ Semantic relationship } & Visual similarity & Manipulation similarity & LSA measure \\
& ratings & ratings & \\
\hline Thematic & $1.76(0.95)$ & $3.71(0.99)$ & $0.36(0.22)$ \\
Specific Function & $2.13(1.42)$ & $4.61(0.92)$ & $0.32(0.30)$ \\
General Function & $2.26(0.92)$ & $4.19(1.43)$ & $0.24(0.15)$ \\
Unrelated similar & $2.64(1.42)$ & $1.41(0.40)$ & $0.001(0.03)$ \\
Unrelated & $1.25(0.26)$ & $1.27(0.26)$ & $0.01(0.22)$ \\
\hline
\end{tabular}

\subsection{Procedure}

All participants provided written informed consent prior to the beginning of the experiment. They were seated in a confortable chair in front of a computer screen $(1920 \times 1080,60 \mathrm{~Hz})$ in a dimly illuminated room. They were informed that after EEG helmet installation and 
familiarization session, they would have to name a series of object pictures. Moreover, they were instructed to fixate on the center of the screen where all events appeared in order to minimize artifacts generated by gaze motion.

\subsubsection{Familiarization Session}

In order to ensure that all participants used the same basic-level names to identify objects, they were submitted to a short familiarization session. During the familiarization session, participants had to name all object pictures presented one at a time on the computer screen. Object written names were then displayed for potential correction.

\subsubsection{Naming Task}

Figure 1 presents the typical trial sequence. First, a fixation cross was displayed for about $1000 \mathrm{~ms}$. Then the prime and target object pictures were presented for $300 \mathrm{~ms}$ in the center of the screen, separated by a blank screen. The duration of the blank screen (Interval Inter-Stimulus or ISI) was either $66 \mathrm{~ms}$ or $250 \mathrm{~ms}$. After target picture presentation, another blank screen was displayed for $700 \mathrm{~ms}$, followed by a question mark. Participants were requested to wait until the appearance of the question mark to name the prime and target pictures in their presentation order. A delayed naming procedure was used in order to prevent EEG signal contamination by mouth movements during naming. Finally, response accuracy was recorded by the experimenter and the question mark was replaced by a hash mark until initiation of the next trial. Instructions stressed the importance of accuracy but did not set any constraint on response times. Only correct trials were considered for further analysis.

The same trial procedure was used for the 180 trials of a naming block: 2 ISI x 2 Prime 
Categories (Related, Unrelated) $\times 3$ Types of Semantic Relation (Thematic, Specific Function, General Function) x 15 target objects. The 180 trials were repeated 4 times in 4 naming blocks, leading to a total of 720 trials. Four trial orders were randomly determined and assigned to each block. Within a block, trial order was fixed for all subjects, but block order was counterbalanced between subjects. Participants could take breaks between blocks.

$<$ Insert Figure 1 $>$ 


\subsection{Data Analysis}

EEG was recorded continuously during the naming task from 128 active electrodes mounted on an elastic cap (10-20 International system Electro-Cap Inc) with an Active Two Biosemi system (Biosemi, Amsterdam, the Netherlands). For each participant, all electrode offsets were kept below $20 \mathrm{mV}$. Moreover, in order to monitor eye movements and blinks, two additional electrodes were placed at lateral canthi and below the eyes. After removing periods with excessive noise artifacts, continuous EEG was digitized at $512 \mathrm{~Hz}$ and filtered offline (1$20 \mathrm{~Hz}$ ) using EEGLAB software (Delorme \& Makeig, 2004), and recalculated to mastoid reference. Moreover, we used ICA-based artifact correction in order to correct blink artifacts (Delorme, Sejnowski, \& Makeig, 2007). Then, the signal was segmented in period of $1800 \mathrm{~ms}$ and time-locked to target picture onset (1000 ms pre-target). At this step, trials presenting wrong naming were removed from analysis (Performance accuracy: 98.15\%). Epochs still contaminated by muscular contractions or an excessive deflection $( \pm 75 \mu \mathrm{V})$ were detected by final visual inspection of the data, and excluded from the averaged Event-Related Potential (ERP) waveforms. Finally, ERPs were computed for each experimental condition using a $200 \mathrm{~ms}$ timewindow before fixation cross as baseline.

Based on ERPs averaged across conditions for the 128 channels, we segmented the signal into four different time windows $(75-160 ; 160-220 ; 220-370$ and $370-510 \mathrm{~ms})$ representing the P1, N1, P3 and N400 components (Figure 2). For each time window, we respectively collapsed ERPs across several electrodes in order to represent maximal activity at each site. Finally, Mean Peak Amplitude for each component was computed by averaging the signal over the respective time windows.

$<$ Insert Figure 2> 
At the neurophysiological level, facilitation for processing objects preceded by related compared to unrelated primes should be reflected by a diminution of the neural response for related compared to unrelated pairs (Bentin, McCarthy, \& Wood, 1985; Helbig et al., 2006; Kiefer et al., 2011). Semantic processing was therefore evaluated through priming effects, which would be visible in the decrease of the amplitude of the component evoked by target objects preceded by semantically related versus unrelated object primes. In other words, the smaller the priming effect amplitude, the greater the brain response to semantically related objects.

Priming effect amplitude was computed for each time window as the difference between semantically related and unrelated object pairs. Semantically unrelated but visually similar object pairs were used as baseline in order to control for the confounded visual similarity between objects that are semantically related. Then, an Analysis of Variance (ANOVA) was conducted on the priming effect amplitude of each component with Type of Semantic Relation (Thematic, Specific Function, General Function), ISI (Short, Long) and Region (Left, Right, Central, depending on the component, see Figure 3) as within-subject factors. The Greenhouse and Geisser (1959) procedure was used to compensate for possible violations of the sphericity assumption. In this case, the corrected degrees of freedom were reported together with the epsilon $(\boldsymbol{\varepsilon})$ and the corrected probability level.

The behavioral studies conducted by Kalenine et al. (2012) and Pluciennicka et al. (submitted) showed different processing time courses for the 3 types of semantic relations. Although the paradigms used are very different and hardly comparable, behavioral results showed a delay of about $200 \mathrm{~ms}$ between thematic and general function similarity relation 
activation during object identification among distractors. Based on these results, two lines of results were expected in the present EEG-priming paradigm.

a) On earlier components (e.g., P1 and/or N1), we predicted a main effect of the Type of Semantic Relation on the amplitude of the priming effect, alone or in interaction with region. Since the Specific Function condition exhibited an intermediate behavioral pattern, we specifically expected a gradation of priming effect amplitude, with Thematic condition < Specific Function condition $<$ General Function condition. Such a gradation was tested using linear (expected significant) and quadratic (expected non significant) orthogonal contrasts defined a priori. Priming effects would be largely reduced, if not absent, in the General Function condition.

b) on later components (e.g., P3 and/or N400), we expected the effect of the Type of Semantic Relation to interact with ISI, alone or in interaction with Region. With limited prime processing time, i.e. with the shorter ISI of $66 \mathrm{~ms}$ (Kiefer et al., 2011), we predicted the same pattern of results as for earlier components, namely graded priming effects, with Thematic condition < Specific Function condition < General Function condition. In contrast, with longer prime processing time (i.e. with the longer ISI of $250 \mathrm{~ms}$ ), the gradual pattern was expected to change dramatically. We hypothesized that activation of general function similarity relations would catch up with thematic activation, which may be either still visible or decreasing. Indeed, behavioral results highlighted a crossover in thematic and general function similarity activation curves about $200 \mathrm{~ms}$ after thematic activation started. Thus, we predicted either an inverted gradation, with General Function condition $<$ Specific Function condition $<$ Thematic conditions, or an absence of gradation, if thematic activation has not ended yet.

The existence of graded priming effects was tested using linear (expected significant) and 
quadratic (expected non significant) orthogonal contrasts defined a priori. In addition, the significance of each individual priming effect was verified by comparing priming amplitude (i.e., difference between related and unrelated pairs) with 0 in each condition using t-tests.

$$
<\text { Insert Figure 3> }
$$

\section{Results}

\subsection{P1 component}

As presented on Figure 3 (left ERP plots and upper table), the ANOVA did not show any main effect of ISI or Type of Semantic Relation on P100 priming effect amplitude (respectively $\mathrm{F}_{1,17}=1.69 ; \varepsilon=1 ; \mathrm{p}=0.211$ and $\left.\mathrm{F}_{1.7,28.4}=1.21 ; \varepsilon=.84 ; \mathrm{p}=0.307\right)$. Furthermore, Type of Semantic Relation did not interact with ISI on P100 priming effect amplitude, alone or combined with Region (for both, $\mathrm{F}_{2,34}<1$ ).

\subsection{N1 component}

The ANOVA showed a significant main effect of the Type of Semantic Relation on N1 Priming effect amplitude $\left(\mathrm{F}_{1.9,33.7}=7.21 ; \varepsilon=.99 ; \mathrm{p}=0.003\right)$. However, the Type of Semantic Relation did not interact with ISI, alone or combined with Region (respectively $\mathrm{F}_{1.7,29.4}=1.33, \varepsilon$ $=.86 ; \mathrm{p}=0.277$ and $\mathrm{F}_{1.6,27.2}<1 ; \varepsilon=.80$ ). Moreover, as shown on Figure 3 (right histograms), planned comparisons revealed that, for all regions and intervals collapsed, Thematic Priming effect amplitude was significantly greater than General Function priming effect amplitude (linear contrast: $F_{1,17}=12.80 ; p=0.003$ ), while Specific Function priming effect amplitude did not differ from the average of the priming effects in the two other conditions (quadratic contrast: $\left.\mathrm{F}_{1,17}=2.48, \mathrm{p}=0.134\right)$. This result demonstrates graded Priming effects on the N1 component 
independently from the region and interval considered, with the Thematic condition showing maximal priming and the General Function condition showing minimal Priming.

Moreover, comparison to 0 in each condition indicated that Priming effects reached significance in the Thematic $\left(t_{17}=2.54 ; p=0.021\right)$, but not in the Specific Function $\left(t_{17}=0.59 ; p=0.561\right)$ and General Function condition $\left(\mathrm{t}_{17}=0.36 ; \mathrm{p}=0.722\right)$.

\subsection{P3 component}

The ANOVA showed significant main effects of Type of Semantic Relation and Region on P3 Priming effect amplitude $\left(\mathrm{F}_{1.8,31.4}=4.87 ; \varepsilon=.92 ; \mathrm{p}=0.008\right.$ and $\mathrm{F}_{1.6,28.1}=6.38 ; \varepsilon=.83 ; \mathrm{p}<$ 0.016 , respectively). In addition, the two factors interacted significantly $\left(\mathrm{F}_{2.2,37.4}=5.43 ; \varepsilon=.55\right.$; $p=0.007)$. However, no significant interaction was found between Type of Semantic Relation and ISI, alone or in interaction with Region (respectively $\mathrm{F}_{2,34}<1 ; \varepsilon=.96$ and $\mathrm{F}_{4,68}=1.66 ; \varepsilon=$ $.82 ; \mathrm{p}=0.182)$

As shown on Figure 2 (right histograms), planned comparisons revealed that, for all ISI collapsed, Thematic and General Function priming effects were significantly different in the 3 regions considered (linear contrast in the left: $F_{1,17}=5.94 ; p=0.026$, Central: $F_{1,17}=12.00 ; p=$ 0.003 and Right: $F_{1,17}=6.84 ; p=0.018$ regions). Moreover, the difference between Specific Function priming effect and the average of the priming effects in the two other conditions was not significant in the central region $\left(\mathrm{F}_{1,17}<1, \mathrm{p}=0.974\right)$, but marginally significant in the left and right regions $\left(\mathrm{F}_{1,17}=3.41, \mathrm{p}=0.082\right.$ and $\mathrm{F}_{1,17}=3.25, \mathrm{p}=0.089$, respectively $)$. Thus, graded Priming effects were observed on the P3 component independently from the ISI considered, with the Thematic condition showing maximal Priming and the General Function condition showing minimal priming. This pattern was, however, clearly visible in the central region only.

In addition, comparison to 0 in each condition indicated that Priming effects reached significance 
only for the Thematic condition in the left and central regions (respectively $\mathrm{t}_{17}=2.51 ; \mathrm{p}=0.022$ and $\left.\mathrm{t}_{17}=2.62 ; \mathrm{p}=0.018\right)$, but not in the right region $\left(\mathrm{t}_{17}=1.19 ; \mathrm{p}=0.250\right)$. Priming effects did not reach significance in the two function conditions, regardless of region (all p-values $>0.137$ ). Thus, when we compared to unrelated primes, we observed a Priming effect on the amplitude of the P3 component only for Thematic related pairs, and particularly in the left and central regions.

\subsection{N400 component}

The ANOVA showed significant main effects of Region and Type of Semantic Relation on N400 Priming effect amplitude $\left(\mathrm{F}_{1.2,20}=19.38 ; \varepsilon=.58 ; \mathrm{p}<0.001\right.$ and $\mathrm{F}_{1.9,32.9}=5.29 ; \varepsilon=.96$; $\mathrm{p}=0.011$, respectively). In addition, there was also a significant interaction between Region and Type of Semantic Relation, alone and in combination with ISI $\left(\mathrm{F}_{2.4,40.5}=8.96 ; \varepsilon=.59 ; \mathrm{p}<0.001\right.$ and $\mathrm{F}_{3.2,54.7}=2.84 ; \varepsilon=.80 ; \mathrm{p}=0.042$, respectively).

As shown on Figure 2 (right histograms), planned comparisons revealed graded Priming effects on the N400 component in the short ISI condition. Indeed, in each region, Thematic and General Function priming effects were significantly (or marginally) different (linear contrast in the left: $F_{1,17}=4.21 ; p=0.055$, Central: $F_{1,17}=11.22 ; p=0.004$ and Right: $F_{1,17}=3.01 ; p=0.101$ ), while Specific Function Priming effects did not differ from the average of the Priming effects in the two other conditions (for all regions, quadratic contrast: $\mathrm{F}_{1,17}<1$ ).

In the longer ISI condition, planned comparison revealed that Thematic and General Function Priming effects were never significantly different (linear contrast in the left: $\mathrm{F}_{1,17}=1.25 ; \mathrm{p}=$ 0.279, Central: $F_{1,17}=4.25 ; p=0.055$ and Right: $\left.F_{1,17}=0.15 ; p=0.707\right)$, and Specific Function priming effects did not differ from the average of the Priming effects in the two other conditions (quadratic contrast in the left: $F_{1,17}=1.84 ; p=0.192$, Central: $F_{1,17}=0.03 ; p=0.858$ and Right: $\mathrm{F}_{1,17}=3.67 ; \mathrm{p}=0.072$ ). Thus, the Priming effect gradation highlighted in the shorter ISI 
condition (Thematic $>$ Specific Function $>$ General Function) disappeared in the long ISI condition.

In the shorter ISI condition, comparisons to 0 in each condition indicated, Priming effects reached significance only in the Thematic (For left, $\mathrm{t}_{17}=2.32 ; \mathrm{p}=0.032$, and central $\mathrm{t}_{17}=4.54 ; \mathrm{p}$ $=0.001$ but not for right region $\mathrm{t}_{17}=1.44 ; \mathrm{p}=0.169$ ) and Specific Function (For central $\mathrm{t}_{17}=$ $3.29 ; \mathrm{p}=0.005$ but not for left and right regions; $\mathrm{t}_{17}=1.65 ; \mathrm{p}=0.115$, and $\mathrm{t}_{17}=0.53 ; \mathrm{p}=0.603$, respectively) conditions. Priming effects were not significant in the General Function condition $\left(\right.$ all $\left.\mathrm{t}_{17}<1\right)$.

By contrast, in the longer ISI condition, Priming effects reached significance in almost all situations. Indeed, N400 Priming effect was systematically observed for Thematic (For left, $\mathrm{t}_{17}=$ 4.15; $\mathrm{p}=0.001$, central, $\mathrm{t}_{17}=5.65 ; \mathrm{p}=0.001$ and right region $\left.\mathrm{t}_{17}=3.31 ; \mathrm{p}=0.005\right)$ and General Function conditions (For left, $\mathrm{t}_{17}=3.37 ; \mathrm{p}=0.005$, central, $\mathrm{t}_{17}=3.28 ; \mathrm{p}=0.005$ and right region $\left.\mathrm{t}_{17}=3.34 ; \mathrm{p}=0.005\right)$. In the Specific function condition, Priming effects were significant in the left $\left(\mathrm{t}_{17}=2.54 ; \mathrm{p}=0.021\right)$ and central $\left(\mathrm{t}_{17}=4.01 ; \mathrm{p}=0.001\right)$ regions, but not in the right region $\left(\mathrm{t}_{17}=1.29 ; \mathrm{p}=0.211\right)$. Overall, comparisons to 0 indicated that when the time between prime and target was limited (shorter ISI), N400 Priming effects were visible for Thematic and Specific conditions (particularly in the central region), whereas with longer ISI, N400 priming effects were obvious for the three Types of Semantic Relation (particularly in the left and central regions).

\subsection{Source visualization}

Finally, in order to visualize the probable generators of the four ERP components of interest, source localization was performed using a distribute source modeling (wMNE, minimum norm source estimates; Hauk, 2004) implemented in Brainstorm (Tadel, Baillet, Mosher, Pantazis, \& 
Leahy, 2011). After the creation of the BEM head model (Gramfort, Papadopoulo, Olivi, \& Clerc, 2010; Kybic et al., 2005), sources were computed for the grand average ERP at each time window (P1, N1, P3 and N400), irrespective of condition (Figure 4, left part) and, as an example, separately for Thematic and General Function conditions at N1 (Figure 4, right part), when the type semantic relation began to generate differences on the ERP. The network involved in object semantic processing from target object onset is largely distributed and includes regions of the occipital cortex, posterior temporo-parietal cortex, and lateral prefrontal cortex, bilaterally. Note that because of the priming paradigm, most regions are already visible from P1, when late prime processing merges with early target processing. At N1, the sources for the two extreme conditions, Thematic and General Function, look relatively similar. Slight differences may be noted, however. Relevant for the current study, at about 200ms of target object processing, regions of the posterior tempo-parietal cortex (red circles) seem more represented in the Thematic than General Function condition.

$<$ Insert Figure 4>

\section{Discussion}

Following behavioral findings showing differences in implicit processing time course of three types of semantic relations involving object "functional" knowledge (Kalénine, Mirman, Middleton, et al., 2012; Pluciennicka et al., submitted), the main goal of the present work was to investigate whether similar time course differences could be highlighted at the neurophysiological level. Findings in this direction would support the existence of distinct cognitive and neural semantic mechanisms during object processing, which would impact different stages of manipulable artifact object visual identification. A protocol combining 
semantic priming with neurophysiological recordings was used (Chen et al., 2014; Kiefer et al., 2011). By manipulating the type of semantic relation and the interval between prime and target object pictures, we could assess fine temporal differences in object semantic processing

The present study highlights two main results: First, we found that ERP components occurring both prior (i.e. N1 and P3) and during (i.e. N400) classical semantic integration were sensitive to the type of semantic relation. On N1 and P3 components, semantic priming was observed for thematic relations only. On N400 component, the type of semantic relation interacted with ISI, and semantic priming was found in all conditions following longer ISI only. In the following sections, we will argue that the ERP differences observed between semantic conditions are two consequences of the different temporal dynamics of thematic, specific function similarity and general function similarity relation processing.

\subsection{Differences in ERP amplitude between semantic conditions are not related to visual similarity or overall semantic relatedness}

Priming effects between related objects are known to be sensitive to visual similarity and overall semantic relatedness between objects (Hutchison, 2003; McRae et al., 2005; van Lier, Leeuwenberg, \& van der Helm, 1997). Accordingly, these two potential confounded factors were carefully controlled (see section 2.1). Overall semantic relatedness measures obtained from Latent Semantic Analysis were kept equivalent between conditions. Thus, we can be fairly confident that priming effect differences between the three types of semantic relations are not due to variations in overall semantic relatedness. In addition, the impact of visual similarity on priming effects was taken into account by introducing an unrelated but visually similar prime, which was visually similar to the target object in terms of color or shape. Visual similar primes 
served as baseline, and were compared to semantically related primes in the computation of semantic priming effects. It is therefore reasonable to believe that amplitude differences between conditions on the N1, P3, and N400 components do not only reflect differences in visual similarity processing.

\subsection{Differences in ERP amplitude reflect time course differences in thematic, specific} function and general function similarity relation processing

The pattern of priming effects observed on ERP amplitudes provides two consistent and complementary pieces of evidence in support of distinct processing time courses for thematic, specific function similarity, and general function similarity relations.

\subsubsection{Early components are only sensitive to thematic priming}

First, graded semantic priming effects were found on N1 and P3 components. Compared to semantically unrelated but visually similar primes, a reduction of the amplitude of the N1 and P3 components was observed when target objects were preceded by thematically related primes (e.g., wood-saw < feather-saw). Conversely, no similar effect was observed when prime and target objects shared a general function similarity (e.g., knife-saw $=$ feather-saw). An intermediate pattern of results was visible when prime and target objects shared a specific function similarity (e.g., wood-saw $<$ axe-saw $<$ knife-saw $=$ feather-saw). In fact, priming effects reached significance on N1 and P3 components in the Thematic condition only. Selective Thematic priming on those early components indicates that thematic relations are processed before functional similarity relations when identifying object pictures.

One could find it surprising that semantic effects were observed on early ERP components. Classically, semantic processing has been observed on the N400 component 
occurring around $400 \mathrm{~ms}$ after stimulus onset (Deacon et al., 2000; Eddy et al., 2006; Kutas \& Iragui, 1998; McPherson \& Holcomb, 1999). However, semantic priming effects related to manipulation similarity have been already documented on components as early as P1, in addition to N400 component (Kiefer et al., 2011). Those early and late semantic priming effects on ERP have been claimed to reflect the involvement of both slow and fast pathways on object recognition. The fast pathway may underlie the early influence of primed features on target visual feature extraction, a processing stage that has been classically associated with P1 (Compton, Grossenbacher, Posner, \& Tucker, 1991; Mangun \& Hillyard, 1991). Templates of probable ERP sources observed at early and late stages of target processing look overall similar with, for example, frontal sources already visible from P1, supporting the early influence of primes on target processing in our paradigm. Although the observation of early semantic effects may be specific to priming procedures, it is interesting to note that they have been previously associated to activation of action representations during object semantic processing. Thus, in our study, one may speculate that thematic priming effects on N1 and P3 components reflect the fast activation of object use representations. The few source variations between Thematic and General Function conditions at N1, which are particularly visible in the posterior temporoparietal cortex, are consistent with this interpretation. This phenomenon would impact the first stages of object recognition, which typically occur within those latencies (Hauk et al., 2007; Johnson \& Olshausen, 2003; Liu, Harris, \& Kanwisher, 2002; Thorpe, Fize, \& Marlot, 1996). Interestingly, Hata et al. (Hata, Homae, \& Hagiwara, 2013) have also reported greater priming effects for artifact than natural object concepts on ERPs about $150 \mathrm{~ms}$ after target onset. Therefore, semantic priming effects on early components might be a marker of action-based semantic priming. Nevertheless, the exact latency from which early semantic priming should be 
visible remains difficult to anticipate (e.g., P1 in Kiefer et al., 2011, N1 in the present study). We will go back to this point when discussing the possible parallel between semantic relations and action representations.

\subsubsection{Late $\mathbf{N} 400$ component is modulated by both semantic condition and ISI}

The second type of effects observed focused on the latter N400 component, known to be involved in semantic processing, in particular detection of semantic deviation (Chen et al., 2014; Deacon et al., 2000; Eddy et al., 2006; Kutas \& Iragui, 1998; McPherson \& Holcomb, 1999). Critically, the priming effect observed on N400 component was sensitive to both semantic priming condition and interval between prime and target. The gradation of semantic priming effects on N400 component with Thematic $>$ Specific Function $>$ General Function was visible in the short interval condition (66 ms) only. In contrast, in the long interval condition ( $250 \mathrm{~ms})$, priming effects did not differ between semantic conditions, and reached significance for the 3 Types of Relation. Sensitivity to ISI provides a second argument in favor of different temporal dynamics for thematic, specific function and general function similarity relation processing at the neural level. After limited processing of the prime object $(366 \mathrm{~ms}$, including $300 \mathrm{~ms}$ target presentation and $66 \mathrm{~ms} \mathrm{ISI),} \mathrm{maximal} \mathrm{priming} \mathrm{was} \mathrm{obtained} \mathrm{for} \mathrm{thematic} \mathrm{relations} \mathrm{that} \mathrm{were}$ behaviorally processed within the shortest time (Kalénine, Mirman, Middleton, et al., 2012; Pluciennicka et al., submitted). When the ISI increased $(550 \mathrm{~ms}$, including $300 \mathrm{~ms}$ target presentation and $250 \mathrm{~ms}$ ISI), and allocated about 200 additional milliseconds to prime processing, priming effects emerged for general function similarity relations, which were processed about $200 \mathrm{~ms}$ later in the previous eye-tracking experiments.

Taken together, these findings corroborated and extend previous observations by highlighting activation of both thematic and functional similarity relations during object 
processing (Cree \& McRae, 2003; Kalénine, Mirman, Middleton, et al., 2012; Lin \& Murphy, 2001; Moss et al., 1995; Pluciennicka et al., submitted). More importantly, they demonstrate gradual latencies for thematic relations, specific function similarity relations, and general function similarity relation activation at the neurophysiological level. The findings reported here may contribute to two parallel theoretical developments in the domain of object semantics. First, they support the existence of several semantic mechanisms and second, they suggest a possible parallel between action and semantic relations between objects..

\subsection{Graded involvement of complementarity and similarity mechanisms in object semantics}

Our findings provide further evidence in favor of distinct functional and neuroanatomical semantic mechanisms (Kalénine et al., 2009; Mirman \& Graziano, 2012; Schwartz et al., 2011; Wisniewski \& Bassok, 1999). On the one hand, there would be a fast semantic mechanism based on processing complementarity between objects, that could impact object processing from the initial stages of object identification (early ERP components), and would involve the posterior temporo-parietal cortex. On the other hand, there would be another semantic mechanism based on similarity processing between object attributes, which would involve more anterior frontotemporal regions and affect later, post-identification object processing stages (i.e. N400 component) when the attributes compared are not only perceptual As visible on the source localization figure and previously highlighted in fMRI (Kalénine et al. 2009), both mechanisms may recruit the two hemispheres. Nevertheless, the findings from Schwartz et al. (2011) in left stroke patients and the present ERP differences, which were more systematically observed in left and central regions, suggest that the distinction may be more pronounced in the left hemisphere. 
Importantly, the weighting of the two mechanisms in semantic processing may depend on many factors, including the type of semantic relation (Kalénine, Mirman, \& Buxbaum, 2012), which may explain why graded semantic effects have been documented on several occasions.

\subsection{Parallel between semantic relations and action representations}

The temporal hierarchy of thematic, specific function, and general function relation processing observed at the neural level may reflect differences in action representation involvement in semantic processing of manipulable artifacts.

A first possibility is that thematic relations rely on action representations while functional similarity relations do not, or to a lower extent, or indirectly later in processing as a result of activation of other types of representations. Selective involvement of action representations in thematic relations would be visible during target object processing from N1. However, in Kiefer et al. (2011)'s study, activation of action representations during manipulation similarity priming was visible from P1. How could we explain this timing discrepancy? We can imagine that actions representations that impact P1 and N1 are not at the same level. Several authors have argued for hierarchical levels of action representations (Cooper \& Shallice, 2006; Grafton \& Hamilton, 2007; Hamilton \& Grafton, 2008, 2009; Kalénine, Shapiro, Flumini, Borghi, \& Buxbaum, 2014; Ortigue, Sinigaglia, Rizzolatti, \& Grafton, 2010; van Elk, Bousardt, Bekkering, \& van Schie, 2012; van Elk, van Schie, \& Bekkering, 2014). For instance, Grafton and Hamilton (2007) have distinguished a kinematic level that codes movement parameters (e.g., close the fingers in particular configuration), a goal-object level that codes object target (e.g., grasp the cup), and an outcome level that codes action expected results (e.g., drink coffee). Manipulation similarity processing between objects may involve action representations at the kinematic levels, which would be visible earlier on visual feature extraction processing stages. Thematic relation 
processing may correspond to the goal-object level of action representations. Using EEG during static action picture perception, Ortigue et al. (2010) and van Elk et al. (2012) showed goal-level representation activation between 200 and 400 milliseconds after picture onset, which correspond to when thematic and functional similarity relation processing differentiated in our study (N1 and P3).

Considering this putative action representation hierarchy, another possibility is that thematic relations rely on action representations at the goal-object level, while functional similarity relations rely on higher levels of action representations (e.g. outcome levels) that would impact late stages of object processing (e.g., N400). The parallel between levels of action representations and various types of semantic relations between manipulable artifacts may be an interesting direction for future research.

\section{Acknowledgments}

This work was funded by the French National Research Agency (ANR-11-PDOC-0014, ANR11-EQPX-0023) and also supported by European funds through the program FEDER SCVIrDIVE. Portions of this research were presented at the 2014 annual meeting of the Cognitive Science Society.

\section{References}

Beauchamp, M. S., \& Martin, A. (2007). Grounding object concepts in perception and action: Evidence from fMRI studies of tools. Cortex, 43, 461-468. 
Bentin, S., McCarthy, G., \& Wood, C. C. (1985). Event-related potentials, lexical decision and semantic priming. Electroencephalography and Clinical Neurophysiology, 60(4), 343-55.

Boronat, C. B., Buxbaum, L. J., Coslett, H. B., Tang, K., Saffran, E. M., Kimberg, D. Y., \& Detre, J. A. (2005). Distinctions between manipulation and function knowledge of objects: evidence from functional magnetic resonance imaging. Brain Research. Cognitive Brain Research, 23(2-3), 361-73.

Buxbaum, L. J., \& Saffran, E. M. (2002). Knowledge of object manipulation and object function: Dissociations in apraxic and nonapraxic subjects. Brain and Language, 82, 179-199.

Buxbaum, L. J., Shapiro, A. D., \& Coslett, H. B. (2014). Critical brain regions for tool-related and imitative actions: a componential analysis. Brain : A Journal of Neurology, 137(Pt 7), $1971-85$.

Canessa, N., Borgo, F., Cappa, S. F., Perani, D., Falini, A., Buccino, G., ... Shallice, T. (2008). The different neural correlates of action and functional knowledge in semantic memory: an FMRI study. Cerebral Cortex, 18(4), 740-51.

Chen, Q., Ye, C., Liang, X., Cao, B., Lei, Y., \& Li, H. (2014). Automatic processing of taxonomic and thematic relations in semantic priming - Differentiation by early N400 and late frontal negativity. Neuropsychologia, 64, 54-62.

Compton, P. E., Grossenbacher, P., Posner, M. I., \& Tucker, D. M. (1991). A cognitiveanatomical approach to attention in lexical access. Journal of Cognitive Neuroscience, 3(4), 304-12. 
Cooper, R. P., \& Shallice, T. (2006). Hierarchical schemas and goals in the control of sequential behavior. Psychological Review, 113(4), 887-916; discussion 917-31.

Cree, G. S., \& McRae, K. (2003). Analyzing the factors underlying the structure and computation of the meaning of chipmunk, cherry, chisel, cheese, and cello (and many other such concrete nouns). Journal of Experimental Psychology. General, 132, 163-201.

Deacon, D., Hewitt, S., Yang, C.-M., \& Nagata, M. (2000). Event-related potential indices of semantic priming using masked and unmasked words: evidence that the N400 does not reflect a post-lexical process. Cognitive Brain Research, 9(2), 137-146.

Delorme, A., \& Makeig, S. (2004). EEGLAB: an open source toolbox for analysis of single-trial EEG dynamics including independent component analysis. Journal of Neuroscience Methods, 134(1), 9-21.

Delorme, A., Sejnowski, T., \& Makeig, S. (2007). Enhanced detection of artifacts in EEG data using higher-order statistics and independent component analysis. NeuroImage, 34(4), $1443-1449$.

Eddy, M., Schmid, A., \& Holcomb, P. J. (2006). Masked repetition priming and event-related brain potentials: a new approach for tracking the time-course of object perception. Psychophysiology, 43(6), 564-8.

Estes, Z., Golonka, S., \& Jones, L. L. (2011). Thematic Thinking. The Apprehension and Consequences of Thematic Relations. Psychology of Learning and Motivation - Advances in Research and Theory (Vol. 54, pp. 249-294). 
Estes, Z., \& Jones, L. L. (2009). Integrative priming occurs rapidly and uncontrollably during lexical processing. Journal of Experimental Psychology. General, 138(1), 112-30.

Farah, M. J., \& McClelland, J. L. (1991). A computational model of semantic memory impairment: modality specificity and emergent category specificity. Journal of Experimental Psychology. General, 120, 339-357.

Garcea, F. E., \& Mahon, B. Z. (2012). What is in a tool concept? Dissociating manipulation knowledge from function knowledge. Memory \& Cognition, 40(8), 1303-13.

Garrard, P., Ralph, M. A., Hodges, J. R., \& Patterson, K. (2001). Prototypicality, distinctiveness, and intercorrelation: Analyses of the semantic attributes of living and nonliving concepts. Cognitive Neuropsychology, 18, 125-174.

Grafton, S. T., \& Hamilton, A. F. de C. (2007). Evidence for a distributed hierarchy of action representation in the brain. Human Movement Science, 26(4), 590-616.

Gramfort, A., Papadopoulo, T., Olivi, E., \& Clerc, M. (2010). OpenMEEG: opensource software for quasistatic bioelectromagnetics. Biomedical Engineering Online, 9, 45.

Hamilton, A. F. de C., \& Grafton, S. T. (2008). Action outcomes are represented in human inferior frontoparietal cortex. Cerebral Cortex (New York, N.Y. : 1991), 18(5), 1160-8.

Hamilton, A. F. de C., \& Grafton, S. T. (2009). Repetition suppression for performed hand gestures revealed by fMRI. Human Brain Mapping, 30(9), 2898-906. 
Hare, M., Jones, M., Thomson, C., Kelly, S., \& McRae, K. (2009). Activating event knowledge. Cognition, 111(2), 151-67.

Hata, M., Homae, F., \& Hagiwara, H. (2013). Semantic categories and contexts of written words affect the early ERP component. Neuroreport, 24(6), 292-7.

Hauk, O. (2004). Keep it simple: A case for using classical minimum norm estimation in the analysis of EEG and MEG data. NeuroImage, 21(4), 1612-1621.

Hauk, O., Patterson, K., Woollams, A., Cooper-Pye, E., Pulvermüller, F., \& Rogers, T. T. (2007). How the camel lost its hump: the impact of object typicality on event-related potential signals in object decision. Journal of Cognitive Neuroscience, 19(8), 1338-53.

Helbig, H. B., Graf, M., \& Kiefer, M. (2006). The role of action representations in visual object recognition. Experimental Brain Research. Experimentelle Hirnforschung. Expérimentation Cérébrale, 174(2), 221-8.

Hutchison, K. A. (2003). Is semantic priming due to association strength or feature overlap? A microanalytic review. Psychonomic Bulletin \& Review, 10(4), 785-813.

Johnson, J. S., \& Olshausen, B. A. (2003). Timecourse of neural signatures of object recognition. Journal of Vision, 3(7), 499-512.

Johnson-Frey, S. H. (2004). The neural bases of complex tool use in humans. Trends in Cognitive Sciences, 8(2), 71-8. doi:10.1016/j.tics.2003.12.002 
Kalénine, S., \& Bonthoux, F. (2008). Object manipulability affects children's and adults' conceptual processing. Psychonomic Bulletin \& Review, 15(3), 667-72.

Kalénine, S., Mirman, D., \& Buxbaum, L. J. (2012). A combination of thematic and similaritybased semantic processes confers resistance to deficit following left hemisphere stroke. Frontiers in Human Neuroscience, 6, 106.

Kalénine, S., Mirman, D., Middleton, E. L., \& Buxbaum, L. J. (2012). Temporal dynamics of activation of thematic and functional knowledge during conceptual processing of manipulable artifacts. Journal of Experimental Psychology. Learning, Memory, and Cognition, 38(5), 1274-95.

Kalénine, S., Peyrin, C., Pichat, C., Segebarth, C., Bonthoux, F., \& Baciu, M. (2009). The sensory-motor specificity of taxonomic and thematic conceptual relations: a behavioral and fMRI study. NeuroImage, 44(3), 1152-62.

Kalénine, S., Shapiro, A. D., Flumini, A., Borghi, A. M., \& Buxbaum, L. J. (2014). Visual context modulates potentiation of grasp types during semantic object categorization. Psychonomic Bulletin \& Review, 21(3), 645-51.

Kellenbach, M. L., Brett, M., Patterson, K., Gee, G., Bisbrown-chippendale, R., Donovan, T., ... Kellenbach, M. (2003). Actions speak louder than functions: The importance of manipulability and Action in tool representation. Journal of Cognitive Neuroscience, 15(1), $30-46$. 
Kiefer, M., Sim, E., Helbig, H., \& Graf, M. (2011). Tracking the Time Course of Action Priming on Object Recognition: Evidence for Fast and Slow Influences of Action on Perception. Journal of Cognitive Neuroscience, 23(8), 1864-74.

Kutas, M., \& Iragui, V. (1998). The N400 in a semantic categorization task across 6 decades. Electroencephalography and Clinical Neurophysiology, 108(5), 456-71.

Kybic, J., Clerc, M., Abboud, T., Faugeras, O., Keriven, R., \& Papadopoulo, T. (2005). A common formalism for the integral formulations of the forward EEG problem. IEEE Transactions on Medical Imaging, 24(1), 12-28.

Labeye, E., Oker, A., Badard, G., \& Versace, R. (2008). Activation and integration of motor components in a short-term priming paradigm. Acta Psychologica, 129, 108-111.

Lee, C., Middleton, E., Mirman, D., Kalénine, S., \& Buxbaum, L. J. (2013). Incidental and context-responsive activation of structure- and function-based action features during object identification. Journal of Experimental Psychology. Human Perception and Performance, 39(1), 257-70.

Lin, E. L., \& Murphy, G. L. (2001). Thematic relations in adults' concepts. Journal of Experimental Psychology. General, 130(1), 3-28.

Liu, J., Harris, A., \& Kanwisher, N. (2002). Stages of processing in face perception: an MEG study. Nature Neuroscience, 5(9), 910-6. 
Mangun, G. R., \& Hillyard, S. A. (1991). Modulations of sensory-evoked brain potentials indicate changes in perceptual processing during visual-spatial priming. Journal of Experimental Psychology. Human Perception and Performance, 17(4), 1057-74.

McPherson, W. B., \& Holcomb, P. J. (1999). An electrophysiological investigation of semantic priming with pictures of real objects. Psychophysiology, 36(1), 53-65.

McRae, K., Cree, G. S., Seidenberg, M. S., \& McNorgan, C. (2005). Semantic feature production norms for a large set of living and nonliving things. Behavior Research Methods, Instruments, \& Computers, 37, 547-59.

Mirman, D., \& Graziano, K. M. (2012). Individual differences in the strength of taxonomic versus thematic relations. Journal of Experimental Psychology. General, 141(4), 601-9.

Moss, H. E., Ostrin, R. K., Tyler, L. K., \& Marslen-Wilson, W. D. (1995). Accessing different types of lexical semantic information: Evidence from priming. Journal of Experimental Psychology: Learning, Memory, and Cognition.

Myung, J. Y., Blumstein, S. E., \& Sedivy, J. C. (2006). Playing on the typewriter, typing on the piano: Manipulation knowledge of objects. Cognition, 98, 223-243.

Oldfield, R. C. (1971). The assessment and analysis of handedness: the Edinburgh inventory. Neuropsychologia, 9, 97-113.

Ortigue, S., Sinigaglia, C., Rizzolatti, G., \& Grafton, S. T. (2010). Understanding actions of others: the electrodynamics of the left and right hemispheres. A high-density EEG neuroimaging study. PloS One, 5(8), e12160. 
Pluciennicka, E., Coello, Y., \& Kalénine, S. (submitted). Development of thematic and functional similarity relation processing during manipulable artifact identification:Evidence from eye-tracking in the Visual World Paradigm.

Pluciennicka, E., Coello, Y., \& Kalénine, S. (in press). Dissociation des relations thématiques et des relations de similarité fonctionnelle entre objets: Données issues des propriétés générées à partir de 100 concepts d'objets fabriqués manipulables. L'année Psychologique.

Rogers, T. T., \& McClelland, J. L. (2004). Semantic cognition: A parallel distributed processing approach. Cambridge, MA, US: MIT Press.

Schwartz, M. F., Kimberg, D. Y., Walker, G. M., Brecher, A., Faseyitan, O. K., Dell, G. S., ... Coslett, H. B. (2011). Neuroanatomical dissociation for taxonomic and thematic knowledge in the human brain. Proceedings of the National Academy of Sciences, 108(20), 8520-4.

Tadel, F., Baillet, S., Mosher, J. C., Pantazis, D., \& Leahy, R. M. (2011). Brainstorm: a userfriendly application for MEG/EEG analysis. Computational Intelligence and Neuroscience, $2011,879716$.

Thorpe, S., Fize, D., \& Marlot, C. (1996). Speed of processing in the human visual system. Nature, 381, 520-522.

Tsagkaridis, K., Watson, C. E., Jax, S. A., \& Buxbaum, L. J. (2014). The role of action representations in thematic object relations. Frontiers in Human Neuroscience, 8, 140. 
Van Elk, M., Bousardt, R., Bekkering, H., \& van Schie, H. T. (2012). Using goal- and griprelated information for understanding the correctness of other's actions: an ERP study. PloS One, 7(5), e36450.

Van Elk, M., van Schie, H., \& Bekkering, H. (2014). Action semantics: A unifying conceptual framework for the selective use of multimodal and modality-specific object knowledge. Physics of Life Reviews, 11(2), 220-50.

Van Lier, R. J., Leeuwenberg, E. L., \& van der Helm, P. A. (1997). In support of hierarchy in object representations. Psychological Research, 60(3), 134-43.

Vingerhoets, G. (2014). Contribution of the posterior parietal cortex in reaching, grasping, and using objects and tools. Frontiers in Psychology, 5, 151.

Warrington, E. K., \& Shallice, T. (1984). Category specific semantic impairments. Brain : A Journal of Neurology, 107 (Pt 3), 829-854.

Watson, C. E., \& Buxbaum, L. J. (2014). Uncovering the architecture of action semantics. Journal of Experimental Psychology. Human Perception and Performance, 40(5), 1832-48.

Wisniewski, E. J., \& Bassok, M. (1999). What makes a man similar to a tie? Stimulus compatibility with comparison and integration. Cognitive Psychology, 39(3-4), 208-38. 


\section{Figure Caption}

\section{Figure 1: Typical trial sequence used during naming task.}

Figure 2: ERP results. On the left panels, averaged ERPs evoked by target objects as a function of the Type of Semantic relation (black solid line for Thematic, grey dashed line for Specific Function, grey dotted line for General Function and black dashed line for Unrelated). The different plots represent the averaged ERPs for the P1, N1, P3 and N400 components in the region where their amplitude was found maximal. Periods represented in grey on the ERP plots correspond to the time windows used to compute the Mean Peak Amplitude for each component. On the right panels, priming effects are presented according to their significant modulating factors: Type of Semantic Relation (all components), Region (P3 and N400 components), and Inter-Stimuli Interval (N400 component). Priming effects correspond to the subtraction between Mean Peak Amplitude evoked by targets primed by semantically related objects, and Mean Peak Amplitude evoked by the same target primed by semantically unrelated (but visually similar) objects. Bars represent standard errors. On the right upper part of the figure, a summary table of the statistical results of the ANOVAs conducted on priming effects is provided. 


\section{Appendix}

Table 2: Approximative English translation of the French stimuli used the present experiment. Thematic relations and specific and general functional similarity relation have been designed from the productions collected in a large property generation study (Pluciennicka et al., in press). Italic terms between brackets provide the function similarity relations shared by prime and target object.

$<$ Insert Table 2> 